\title{
Current techniques in reprogramming cell potency
}

\begin{abstract}
The first successful attempt to reprogram somatic cell into embryonic-like stem cell was achieved on 2006. Since then, it had sparked a race against time to bring this wonderful invention from bench to bedside but it is not easily achieved due to severe problems in term of epigenetic and genomic. With each problem arise, new technique and protocol will be constructed to try to overcome it. This review addresses the various techniques made available to create iPSC with problems hogging down the technique.
\end{abstract}

Keyword: iPSC; Reprogramming; Stem Cell 